\title{
Intelligent HMI and Control for Steering a Powered Wheelchair Using a Raspberry Pi Microcomputer
}

\author{
Malik Haddad \\ School of Mechanical and Design Engineering \\ University of Portsmouth \\ Portsmouth, UK \\ malik.haddad@port.ac.uk \\ Favour Ikwan \\ School of Mechanical and Design Engineering \\ University of Portsmouth \\ Portsmouth, UK \\ favour.ikwan@port.ac.uk \\ Martin Langner \\ Chailey Heritage Foundation / Engineering \\ Chailey Heritage Foundation \\ Chailey, UK \\ mlangner@chf.org.uk
}

\author{
David Sanders \\ School of Mechanical and Design Engineering \\ University of Portsmouth \\ Portsmouth, UK \\ david.sanders@port.ac.uk \\ Mohamad Thabet \\ School of Mechanical and Design Engineering \\ University of Portsmouth \\ Portsmouth, UK \\ mohamad.thabet@port.ac.uk
}

\author{
Alexander Gegov \\ School of Computing \\ University of Portsmouth \\ Portsmouth, UK \\ alexander.gegov@port.ac.uk
}

\begin{abstract}
This paper presents a new technique for controlling powered wheelchairs. A Raspberry Pi microcomputer is used to assist in controlling direction. A Raspberry $\mathrm{Pi}$ is inserted between user input switches and powered wheelchair motors to create a more intelligent Human Machine Interface (HMI). An electronic circuit is created that consists of an ultrasonic sensor array and a set of control relays. The sensors provided information about obstacles surrounding the wheelchair. Python programming language was used to create a code that digitized the output from the user switches and assessed information provided by the ultrasonic sensor array. The code was loaded onto a Raspberry $\mathbf{P i}$ and the Raspberry Pi controlled voltages supplied to the motors. Tests were conducted and results showed that the new system can successfully assist a wheelchair user in avoiding obstacles. The system can be used as an intelligent interface between any input device or sensor system and wheelchair motors.
\end{abstract} Pi; steer

Keywords-direction; avoidance; Python; wheelchair; Raspberry

\section{INTRODUCTION}

Research described here is part of a larger research project to improve mobility and enhance the quality of life of disabled powered wheelchair users by increasing their selfconfidence and reliance [1]. A method is described here that manages steering inputs from users and mixes them with sensors to assist a disabled driver to steer safely.

Seven million citizens in the USA use assistive apparatus and mobility devices. Statistics say that about two citizens use a wheelchair or scooter and another five million use walkers, canes, crutches and other similar passive tools to assist them [2]. About 2.3 million need others to assist them. Osteoarthritis and stroke are the main disabilities recorded for people who use a wheelchair or scooter [3]. A main disorder accompanying a recorded use of a mobility device is osteoarthritis [2]. Disabled users of powered wheelchairs may not have the dexterity required or sufficient mobility due to hand, finger, shoulder, arm or more widespread incapability, and they may not have adequate lower limb strength to propel a manual wheelchair [4].

The bearing and pace of a powered wheelchair is often controlled by a joystick. When a wheelchair user does not have sufficient coordination or cannot use their fingers or hands then alternatives can be used, for example: puff switches, sip-tubes, and head, finger, chin, neck or foot controllers. If a user is blind, has a head injury, physiological or neurological problems, or not enough spacial awareness, then they will not be able to safely steer a wheelchair as they could not safely take any avoiding action. Systems described here can help drivers to steer their wheelchair safely.

Wheelchair controllers are usually open-loop. A driver indicates speed and direction using a transducer, for example levers, switches or joysticks. A wheelchair then tends to move at the preferred speed in the chosen direction. A user can then make corrections to circumvent obstacles. The preferred bearing is mixed with sensor information [5 - 17] to assist the drivers. 
Navigation and steering has been explored [18 - 22] but techniques have typically been local rather than global and obstacles avoidance has been considered [23] using local sensors [24]. Initial wheelchair paths have been calculated that are adapted locally when there are obstructions detected, but they have rarely moved out of the laboratory to positively assist disabled wheelchair users. A new microcomputer system is described here that uses sensors to successfully assist with driving. The new system quickly reacts to objects and tends to stop a user from moving in the direction of an obstacle.

Raspberry Pi microcomputers were considered as reliable powerful computers with compact physical size and affordable price, they were first introduced in 2012 as 2 x models: A and B. In 2014, Model B+ was introduced. It contained numerous small enhancements based on suggestions from users [25]. The research conducted in this paper used Raspberry Pi model B + .

An ultrasonic sensor array was used to detect obstacles in the surrounding of a wheelchair. Powered wheelchair users supplied their desired direction through analogue switches. Python programming language was used to create a code that read users' supplied direction and assessed information provided from the ultrasonic sensor array. Code was loaded to a Raspberry Pi. The Raspberry $\mathrm{Pi}$ and the electronic circuit converted users supplied input from switches into logic levels. The desires of a human driver were traded against distance to nearby obstacles. If there was no obstacle detected in the direction supplied by the user, the Raspberry Pi triggered a set of specific relays that supplied voltages to the wheelchair motor responsible for moving the wheelchair in the desired direction. If an obstacle was detected in the direction supplied by the user, the Raspberry Pi cut-off voltages from the motor responsible for moving the wheelchair in that direction and avoided the obstacle. Obstacle detection range could be digitally adjusted by the intelligent controller from $2 \mathrm{~cm}$ to $500 \mathrm{~cm}$. New processes were simulated before testing in the laboratory using arrays of ultrasonic sensors, Fig. 1 shows an example of powered wheelchair used in the research.

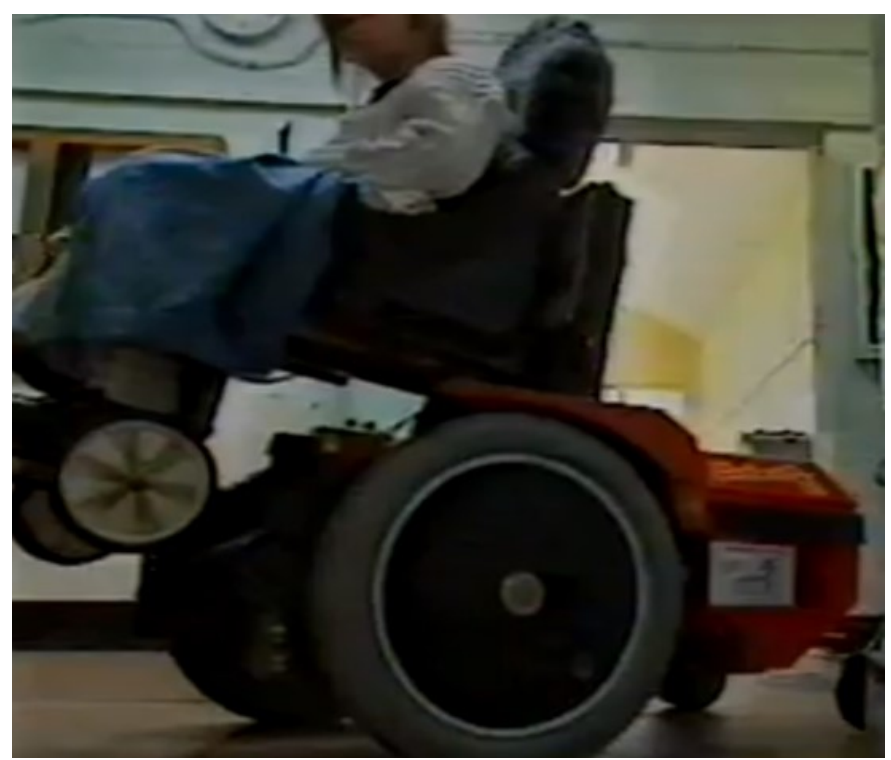

Figure 1. The Bobcat 2 Powered Wheelchair used for the research
An outer fiberglass shell enclosed the wheelchair base and the base itself was made from a steel frame that provided strength and stability. Casters were trailing at the rear and there were two oversized driving wheels at the front. Fig. 1 shows the two large driving-wheels. The wheels are fixed along a mutual axis. Motion, Bearing and Orientation were achieved through driving each of the large wheels independently with a separate independent motor connected to each wheel; drivers could steer by varying electrical current sent to each motor.

Sensors to assist wheelchair users with avoiding items safely were described by Sanders et al. [26], including infrared sensors [27] ultrasonics [28], and structured lighting [29]. Global methods have been awkward to use inside buildings [30] but local systems have been more successful: gyroscopes, odometers, tilt sensors and ultrasonics [31, 32 \& 33]. Camera prices have been dropping, but the processing of the data can be complicated. Computer prices have also dropped, and computers are becoming more powerful [30], so cameras are often used. Despite that, a disabled powered wheelchair driver can often provide the best data about what is required, but reduced visibility or a disability may still reduce that ability [34].

In the work presented here, ultrasonics have been used because they were simple, robust, and low cost [32]. Section 2 briefly explains the sensors, Section 3 presents the intelligent controller and Human Machine Interface (HMI) created to interface the sensors. Section 4 presents results and Section 5 presents discussion and conclusions.

\section{SENSORS}

Sensors used in this research were similar to those presented and used in [35]. They were placed under the footrests [36]. The distance from a detected object was determined from the time that a pulse took to send and reflect back to a receiver(s) [37]. Fig. 2 shows the rays of the sensors and a potential grid that can be created.

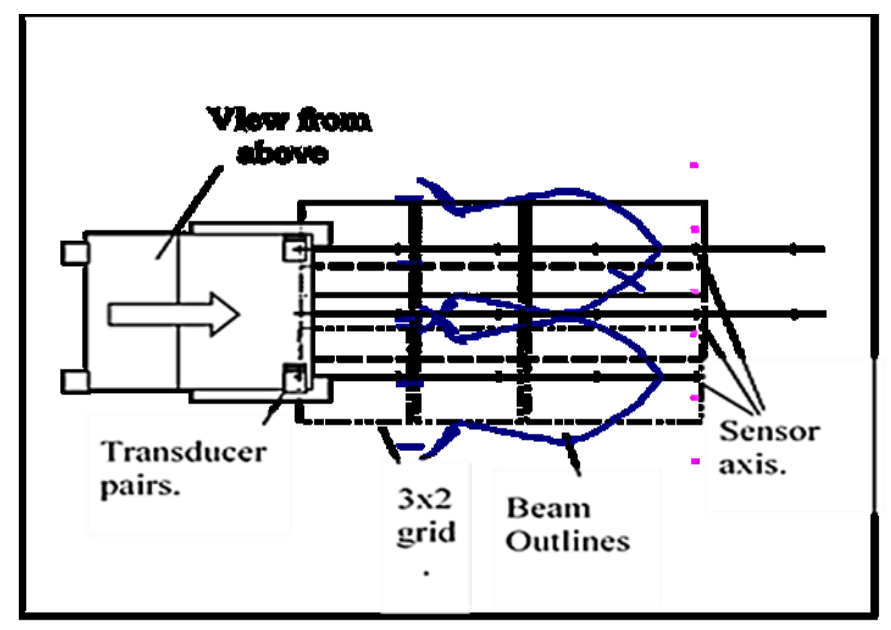

Figure 2. An array created form overlapping ultrasonic rays

If nothing was sensed, then range could be digitally enlarged by generating longer pulses until an object was detected. Warnings could then be provided concerning problems ahead. 
HC SR04 ultrasonic sensors were studied and tested using different objects to create polar plots. Due to their physical structure, these sensors did not suffer from side lobe interference. Objects considered varied in shapes and sizes. The sensors detected small cylindrical object less than $0.65 \mathrm{~cm}$ in diameter up to $15 \mathrm{~cm}$ away. The larger the object, the larger the detection distance. Fig. 3 shows the setting used to test the sensors. Figs. 4-7 shows polar plots for different HC SR04 sensors detecting different objects at different distances.

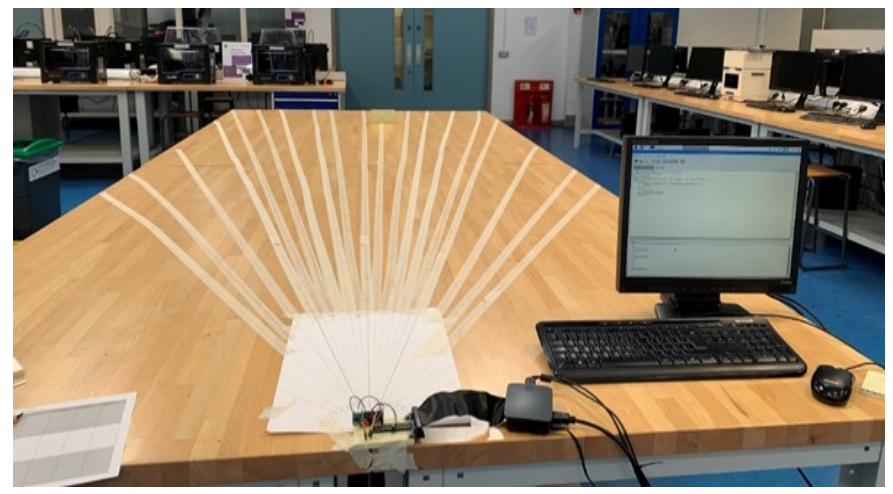

Figure 3. Laboratory setting used to test HC SR04 ultrasonic sensors

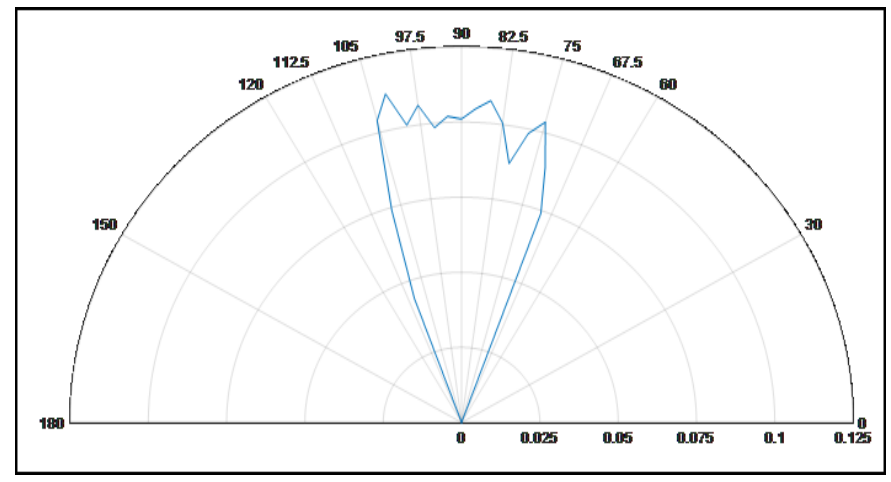

Figure 4. Polar plot for HC SR04 ultrasonic sensor detecting $0.639 \mathrm{~cm}$ cylindrical object

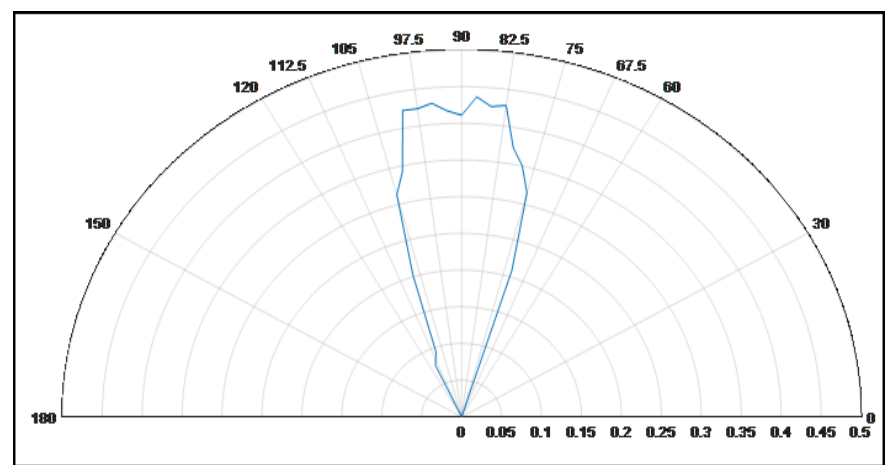

Figure 5. Polar plot for HC SR04 ultrasonic sensor detecting $2.188 \mathrm{~cm}$ cylindrical object

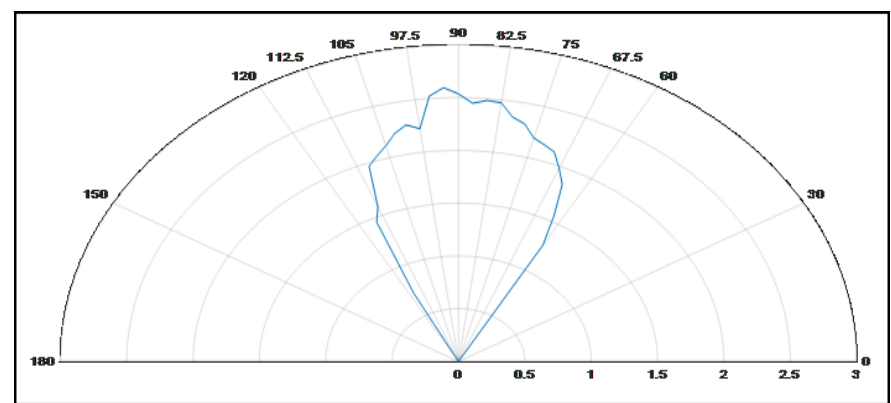

Figure 6. Polar plot for HC SR04 ultrasonic sensor detecting detecting (3.2X9.7) $\mathrm{cm}$ rectangular object

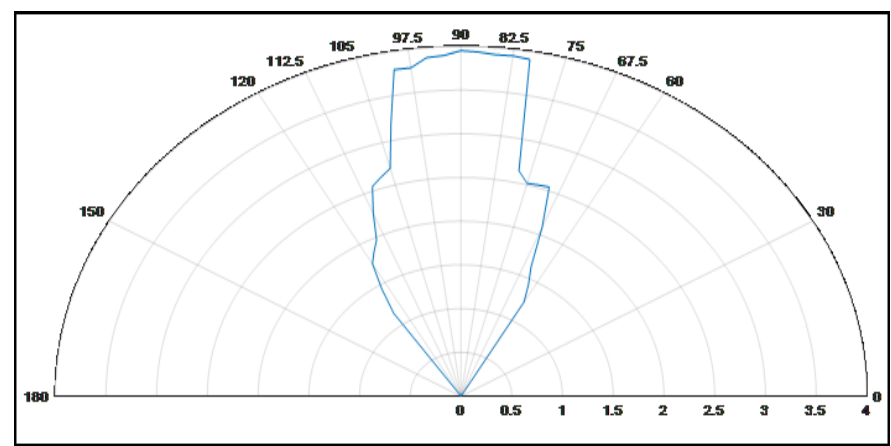

Figure 7. Polar plot for HC SR04 ultrasonic sensor detecting detecting (8X14) $\mathrm{cm}$ rectangular object

A configuration of five ultrasonic sensors was used to create an array, the ultrasonic sensors array was used to assess the wheelchair surroundings. Five HC SR04 ultrasonic sensors were used ( 3 were positioned to the front, 1 to the left and 1 to the right). The sensors provided distance to the nearest obstacle in their detection zone. Python programming language was used to create a program to prevent a wheelchair from driving in the direction of an obstacle if the obstacle was detected at a near distance. The range could be adjusted digitally in the program from $2 \mathrm{~cm}$ to $500 \mathrm{~cm}$. Fig. 8 shows an ultrasonic sensor array connected to a Raspberry Pi (left), a set of user switches (bottom right) and a set of relays that controlled the supplied voltages (middle).

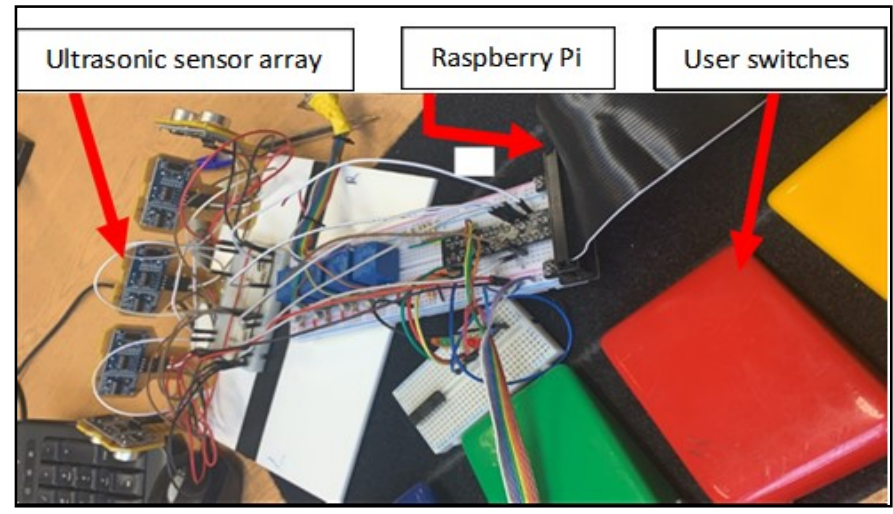

Figure 8. Ultrasonic array connected to a Raspberry Pi microcomputer and user switches 


\section{INTELLIGENT CONTROLLER AND HUMAN MACHINE INTERFACE (HMI)}

An electronic circuit digitized the output of the users' switches. A Raspberry Pi microcomputer was inserted between the users' switches and the wheelchair motors. The Raspberry $\mathrm{Pi}$ could be used for intelligent controller and as an interface with any type of input device, for example a joystick, chin switch, blow switch, head switch or an EEG helmet.

Users' switches were connected to 9 pin D-connector. The following steps were used to digitize the output from the users' switches in order to create the intelligent controller and HMI

1) Voltage reduction $(12 \mathrm{~V} \rightarrow 5 \mathrm{~V})$ : The switches operated two $12 \mathrm{~V}$ DC motors, this voltage would damage a Raspberry Pi. Raspberry Pi operated at $5 \mathrm{~V} \mathrm{DC}$. The output from the input switches was reduced to values compatible with the Raspberry Pi. Output from users' switches (D-connector) were connected to a CD4050BE Hex Buffer as shown in Fig. 9. The buffer reduced the voltage from $12 \mathrm{~V}$ to $5 \mathrm{~V}$ which was compatible with the Raspberry Pi.

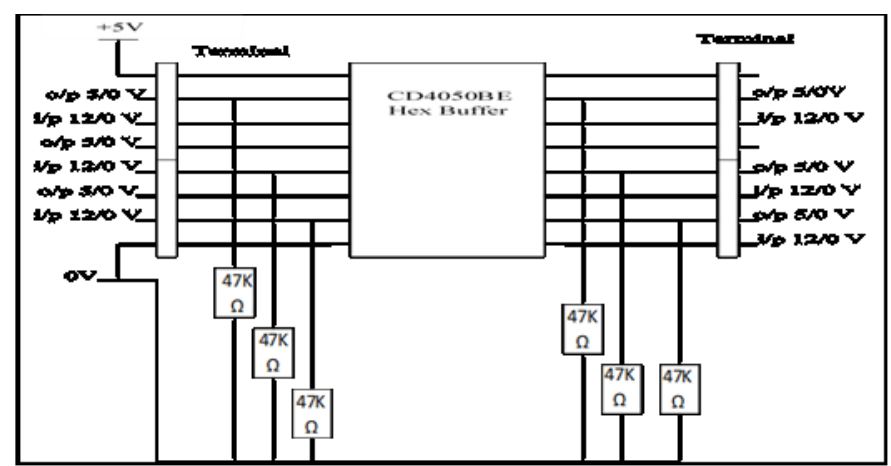

Figure 9. 4050BE Hex Buffer circuit diagram

2) Create a program using Python programming language to read the output from users' switches. If a switch was pressed, the Raspberry Pi and Python code would assign high logic value to a designated pin identified in the program.

3) Regulate output voltage from Raspberry Pi. A Logic high voltage from Raspberry $P i$ was 3.3 volts, this value was not enough to trigger $5 \mathrm{~V} D \mathrm{C}$ relays used to control the function of the wheelchair motors. Common collector circuits were created to drive the $5 \mathrm{~V}$ DC relays. Output from Raspberry Pi were connected to the bases of a set of NPN transistor (BC107), collectors of the transistors were connected to the relay triggers, when logic high was supplied from the Raspberry Pi to a specific transistor base, the collector of that transistor would provide voltage capable of triggering the specific relay responsible for switching the required motor. Fig. 10 shows the circuit diagram of the common collector circuit used. Fig. 11 shows a prototype of the circuits used.

4) Create Python code to control the wheelchair and provide the intelligent HMI.

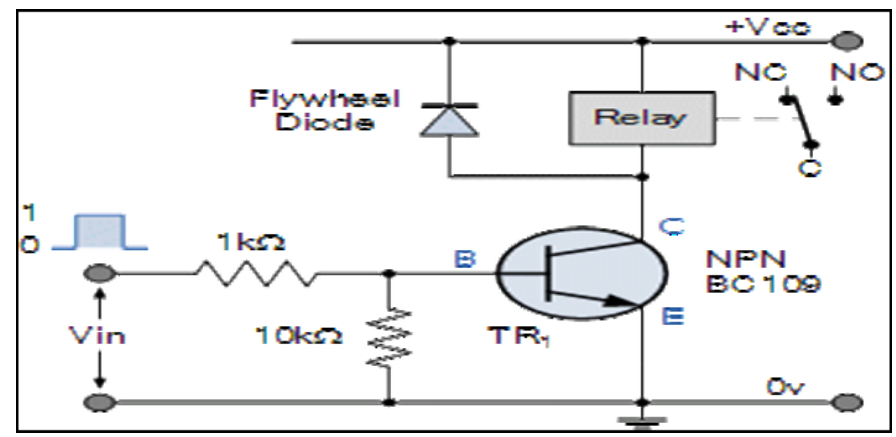

Figure 10. Common Collector circuit diagram

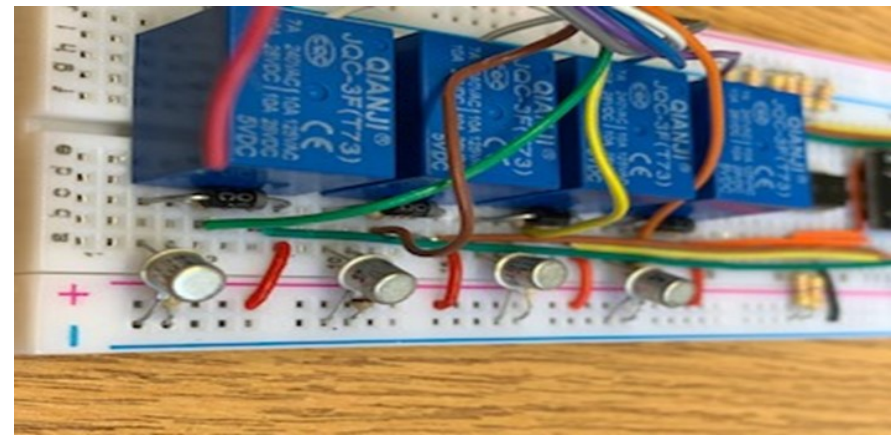

Figure 11. Prototype of the Common Collector circuits used

Since the relay coil could not instantly change current, a flyback diode was used to provide a path for the current when the coil was switched off which prevented a voltage spike from occurring and damaging switch contacts or destroying switching transistors.

Python programs are available from the corresponding author upon request.

\section{RESULTS}

This research used an ultrasonic sensor array to detect the surrounding of a powered wheelchair, digitized the outcome of users' switches and helped wheelchair users in avoiding obstacles.

Pressing a users' switch triggered a specific 5V DC relay that controlled the voltage supplied to the wheelchair motor responsible to move the wheelchair in the desired direction, a simulation with $12 \mathrm{~V}$ bulbs to show the operation of the relays is shown in Figs. 12 and 13.

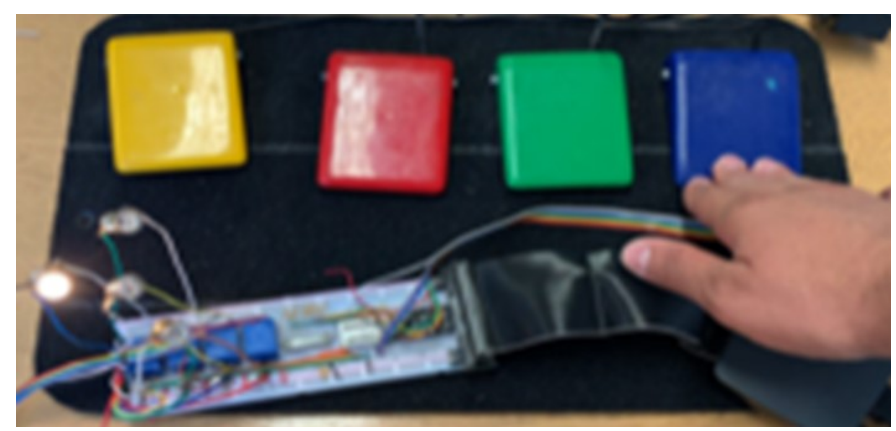

Figure 12. Controlling the output of relays using input switches 


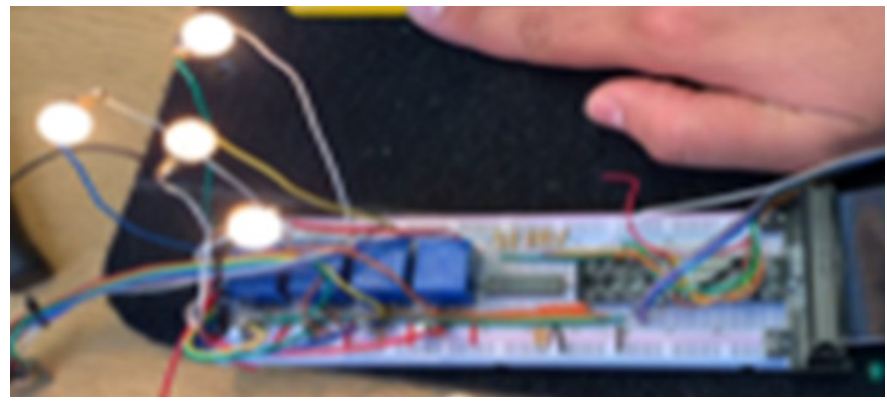

Figure 13. Pressing all input switches controlled the output of all relays

Analogue switches were used to afford an interface linking a disabled user and a wheelchair to regulate bearing and speed. The digitization of the output of the analogue switches combined suggestions from a sensor system and input from a disabled driver and could improve driving by reducing the number of collisions, providing an intelligent route that avoided obstacles and analysed the surrounding of the wheelchair. Human drivers often use their own skill to control their powered wheelchair, but sensors provided a more repeatable and accurate reading, and could compensate for insufficient ability or awareness. The new research combined human driving skill with autonomy and then intervention from the systems if required. When moving through complex or changing surroundings the sensors could provide better information and that led to better decisions being made about new bearings to move to.

Disabled wheelchair drivers could effectively drive their wheelchairs using their input device and the system avoided objects. The sensors ensured that the wheelchair was safe as it moved.

\section{DISCUSSION AND CONCLUSIONS}

The research presented in this paper is part of a broader research concerned in improving mobility and enhance the quality of life of disabled powered wheelchair users by increasing their self-confidence and reliance.

This paper presented a system that could be used as an interface between any user input device and a powered wheelchair motors. The new systems might reduce the need for carers and introduce more autonomy.

The electrical current that drove the motors was created from a mix of the output form the sensors and the input from the disabled driver. With a smaller quantity of obstacles or if the obstacle(s) was far-off, then a driver would not need any assistance. Giving a disabled wheelchair user higher authority in that case assured improved performance. If there were many objects in the way or there were obstructions close to the wheelchair, then the new sensor systems were able to reduce inputs from joysticks or switches in order to prevent a collision from occurring.

The authors are currently studying ways to apply machine learning and other AI techniques to improve this research.

Future work will consider the application of machine learning techniques and aim at collecting user data to predict driving patterns, assess driving skills progress and analyze ability factors denoting the ability of a user to operate their wheelchair. Specifically some decision making algorithms will be investigated [38-40].

Results showed that the new system performed accurately. Systems and methods will be clinically trialed at Chailey Heritage School during a large ERSPC research project [1].

\section{ACKNOWLEDGMENT}

This research was supported by an EPSRC EP/S005927/1 project titled "Using artificial intelligence to share control of a powered-wheelchair between a wheelchair user and an intelligent sensor system". Investigators: Sanders, DA and Gegov, AE. Senior Researcher Haddad MJM.

\section{REFERENCES}

[1] D. R. Parhi, and M. K. Singh, "Rule-based hybrid neural network for navigation of a wheelchair,” P I Mech Eng B-J Eng, vol. 224, pp.11103 $-1117,2009$.

[2] D. A. Sanders, A. Gegov, D. Ndzi, "Knowledge-based expert system using a set of rules to assist a tele-operated mobile robot," Studies in Computational Intelligence, 751, Springer, 2018, pp. 371-392, 2018.

[3] D. A. Sanders, et al. "A Rule-based Expert System to decide on direction and speed of a powered-wheelchair," IEEE Proceedings of the SAI Conference on IntelliSys. London, U.K., pp. 426 - 433, 2018.

[4] D. Sanders, M. Langner, N. Bausch, Y. Huang, S. A. Khaustov, and S. Simandjuntak, "Improving human-machine interaction for a poweredwheelchair driver by using variable-switches and sensors that reduce wheelchair-veer," Intelligent Systems and Applications. Adv. Intell. Syst. Comput, vol. 1038, Springer, pp. 1173-1191, 2019.

[5] O. M. Okonor, A. Gegov, M. Adda, D. Sanders, M. Haddad, and G Tewkesbury, "Intelligent approach to minimizing power consumption in a cloud-based system collecting sensor data and monitoring the status of powered-wheelchairs," Intelligent Systems and Applications. Adv. Intell. Syst. Comput, vol. 1037, Springer, pp. 694-710, 2019.

[6] D. Sanders, O. M. Okonor, M. Langner, S. M. Hassan, S. A. Khaustov, and P. O. Omoarebun, "Using a simple expert system to assist a powered-wheelchair user," Intelligent Systems and Applications. Adv. Intell. Syst. Comput, vol. 1037, Springer, pp. 662-379, 2019.

[7] N. Bausch, P. Shilling, D. Sanders, M. Haddad, O. M. Okonor, and G Tewkesbury, "Indoor location and collision feedback for a poweredwheelchair system using machine learning," Adv. Intell. Syst. Comput, Vol. 1 pp. 721-739, 2019.

[8] G. Tewkesbury, D. Sanders, M. Haddad, N. Bausch, A. Gegov, and O.M. Okonor, "Task programming methodology for poweredwheelchairs," Adv. Intell. Syst. Comput, Vol. 1 pp. 711-720, 2019.

[9] D. Sanders, G. Tewkesbury, H. Parchizadeh, J. J. Robertson, P. O Omoarebun, and M. Malik, "Learning to drive with and without intelligent computer systems and sensors to assist," Adv. Intell. Syst. Comput, vol. 868, Springer, pp. 1171-1181, 2019.

[10] D. Sanders, A. Gegov, M. Haddad, F. Ikwan, D. Wiltshire, and Y. C. Tan, "A rule-based expert system to decide on direction and speed of a powered-wheelchair,” Adv. Intell. Syst. Comput, vol. 868, Springer, pp. 822-838, 2019.

[11] M. Haddad, D. Sanders, N. Bausch, G. Tewkesbury, A. Gegov, and M Hassan Sayed, "Learning to make intelligent decisions using an Expert System for the intelligent selection of either PROMETHEE II or the Analytical Hierarchy Process," Adv. Intell. Syst. Comput, vol. 868, Springer, pp. 1303-1316, 2019.

[12] D. R. Parhi et al. "The stable and precise motion control for multiple wheelchairs", Appl. Soft Comput, vol. 9, no. 2, pp. 477 - 487, 2009.

[13] V. Nguyen et al. "Strategies for Human - Machine Interface in an Intelligent Wheelchair," 35th Annual Int Conf of IEEE-EMBC, Osaka, 
JAPAN. Book Series: IEEE Engineering in Medicine \& Biology Society, 2013, pp. 3638-3641.

[14] M. Haddad, D. Sanders, A. Gegov, A, M. Hassan Sayed, Y. Huang, and M. Al-Mosawi, "Combining multiple criteria decision making with vector manipulation to decide on the direction for a poweredwheelchair," Adv. Intell. Syst. Comput, vol. 1037, Springer, pp. 680693, 2019.

[15] M. Haddad, D. Sanders, G. Tewkesbury, A. Gegov, M. Hassan Sayed, and F. Ikwan, "Initial results from using Preference Ranking Organization METHods for Enrichment of Evaluations to help steer a powered-wheelchair," Adv. Intell. Syst. Comput, vol. 1037, Springer, pp. 648-661, 2019.

[16] D. Sanders, Q. Wang, N. Bausch, Y. Huang, S. A. Khaustov, and I. Popov, "A method to produce minimal real time geometric representations of moving obstacles, Adv. Intell. Syst. Comput, vol. 868, Springer, pp. 881-892, 2019

[17] D. Sanders, A. Gegov, G. Tewkesbury, and R. Khusainov, "Sharing driving between a vehicle driver and a sensor system using trust-factors to set control gains," Adv. Intell. Syst. Comput, vol. 868, Springer, pp. 1182-1195, 2019.

[18] D. A. Sanders et al. "Results from investigating powered-wheelchair users learning to drive with varying levels of sensor support," in Proc SAI Intelligent System, London, U.K. 2017, pp. 241-245.

[19] K. T. Song, and C. C. Chen, "Application of asymmetric mapping for wheelchair navigation using ultrasonic sensors," J. Intell. Wheelchair Syst., vol. 17 , no. 3, pp. 243 - 264, 1996

[20] D. Sanders, M. Langner, and G. Tewkesbury, "Improving wheelchairdriving using a sensor system to control wheelchair-veer and variableswitches as an alternative to digital-switches or joysticks," Ind Rob: An int' jnl, vol. 37, no. 2, pp.151-167, 2010.

[21] S. Lee,"Use of infrared light reflecting landmarks for localization," Ind Rob: An int' jnl, vol. 36, no. 2, pp. 138-145, 2009.

[22] D. Sanders, I. Stott, "A new prototype intelligent mobility system to assist powered-wheelchair users," Ind Rob, vol. 26, no. 6, pp. 466-475, 2009.

[23] J. Larsson, M. Broxvall, and A. Saffiotti, "Laser-based corridor detection for reactive Navigation," Ind Rob: An int' jnl, vol. 35, no. 1, pp. 69-79, 2008

[24] V. Milanes, J. Naranjo, and C. Gonzalez, "Autonomous vehicle based in cooperative GPS and inertial systems," Robotica, vol. 26, pp. 627-633, 2008 .

[25] D. A. Sanders, "Controlling the direction of walkie type forklifts and pallet jacks on sloping ground," Assem. Autom., vol. 28, no. 4, pp. 317 324, 2008.

[26] D. Sanders, "Recognizing shipbuilding parts using artificial neural networks and Fourier descriptors," P I Mech Eng B-J Eng, vol. 223, no. 3, pp. 337-342, 2009.

[27] Y. C. Chang, and Y. Yamamoto, "On-line path planning strategy integrated with collision and dead-lock avoidance schemes for wheeled wheelchair in indoor environments," Ind Rob: An int' jnl, vol. 35, no. 5, pp. 421-434, 2008.

[28] D. Sanders, "Comparing speed to complete progressively more difficult mobile robot paths between human tele-operators and humans with sensor-systems to assist," Assem. Autom., vol. 29, no. 3, pp. 230-248, 2009.

[29] A. Ishizaka, S. and Siraj, "Are multi-criteria decision-making tools useful? An experimental comparative study of three methods," EJOR, 264, 462-471, 2018

[30] D. A. Sanders, "Using self-reliance factors to decide how to share control between human powered-wheelchair drivers and ultrasonic sensors," IEEE Trans Neural Syst Rehabil Eng, vol. 25, no. 8, pp. 1221 $1229,2017$.

[31] D. Sanders, et al. "Tele-operator performance and their perception of system time lags when completing mobile robot tasks," Proc 9th Int Conf on Human Systems Interaction, 2016, pp. 236-242.

[32] K. Raju, and D. Kumar, "Irrigation Planning using Genetic Algorithms," Water Resour Manag, vol. 18, pp. 163 - 176, 2004.

[33] M. Haddad, D. Sanders, and N. Bausch, "Selecting a robust decision making method to evaluate employee performance," Int J Manag Decis Making, vol. 18, no. 4, pp. 333-351, 2019.

[34] T. L. Saaty, "Decision making with the analytic hierarchy process," Int J Serv Sci, vol. 1, no. 1, pp. 83-98, 2008.

[35] A. Ishizaka, and A. Labib, "Analytic hierarchy process and expert choice: Benefits and limitations," Or Insight, vol. 22, no. 4, pp. 201-220, 2009

[36] A. Gegov, N. Gobalakrishnan, and D. A. Sanders, "Rule base compression in fuzzy systems by filtration of non-monotonic rules," J Intell Fuzzy Syst, vol. 27, no. 4, pp. 2029-2043, 2014.

[37] D. A. Sanders et al. "Rule-based system to assist a tele-operator with driving a mobile robot," Lecture Notes in Networks and Systems, 16, Springer, pp. 599-615, 2018.

[38] M. Haddad, and D. Sanders, "Visual representation of stability for three discrete Multi-Criteria Decision Making Methods," J Oper Res Soc, Submitted and Under Review.

[39] M. Haddad, D. Sanders, and G. Tewkesbury, "Selecting a discrete Multiple Criteria Decision Making method to decide on a corporate relocation," Arch Bus Res, vol. 7, no. 5, pp. 48-67, 2019.

[40] M. Haddad, and D. Sanders, "Selecting a best compromise direction for a powered-wheelchair using PROMETHEE," IEEE Trans Neural Syst Rehabil Eng, vol. 27, no. 2, pp. 228-235, 2019.

[41] D. Sanders, D. C. Robinson, M. Hassan Sayed, M. Haddad, A. Gegov, and N. Ahmed, "Making decisions about saving energy in compressed air systems using Ambient Intelligence and Artificial Intelligence," Adv. Intell. Syst. Comput, vol. 869, Springer, pp. 1229-1236, 2018.

[42] D. Sanders, and A. Gegov, "Using artificial intelligence to share control of a powered-wheelchair between a wheelchair user and an intelligent sensor system," EPSRC Project 2019-2022, 2018.

[43] D. A. Sanders, "The modification of pre-planned manipulator paths to improve the gross motions associated with the pick and place task," Robotica, vol. 13, pp. 77-85, 1995.

[44] D. A. Sanders, "Viewpoint - Force sensing," Industrial Robot, vol. 34, p. $177,2007$.

[45] D. Sanders, "Comparing ability to complete simple tele-operated rescue or maintenance mobile-robot tasks with and without a sensor system," Sensor Rev, vol. 30, no. 1, pp. 40-50, 2010. 